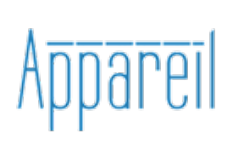

Appareil

1| 2008

Le milieu des appareils

\title{
Philosophie de la nature et artefact
}

La question du « préindividuel »

Jean-Hugues Barthélémy et Vincent Bontems

\section{(2) OpenEdition}

Journals

Édition électronique

URL : http://journals.openedition.org/appareil/72

DOI : 10.4000/appareil.72

ISSN : 2101-0714

Éditeur

MSH Paris Nord

Référence électronique

Jean-Hugues Barthélémy et Vincent Bontems, "Philosophie de la nature et artefact », Appareil [En

ligne], 1 | 2008, mis en ligne le 09 février 2008, consulté le 30 juillet 2020. URL : http://

journals.openedition.org/appareil/72 ; DOI : https://doi.org/10.4000/appareil.72

Ce document a été généré automatiquement le 30 juillet 2020.

\section{(c)}

Appareil est mis à disposition selon les termes de la Licence Creative Commons Attribution - Pas d'Utilisation Commerciale - Pas de Modification 4.0 International. 


\title{
Philosophie de la nature et artefact
}

\author{
La question du « préindividuel » \\ Jean-Hugues Barthélémy et Vincent Bontems
}

Le dialogue qu'a instauré l'œuvre de Bernard Stiegler avec la pensée de Gilbert Simondon se veut une opération de prolongement mais aussi de dépassement, en cela comparable, dans une certaine mesure, au dialogue que Simondon avait lui-même instauré avec Henri Bergson. Or, la nature de ce nouveau dialogue a ceci de spécifique, toutefois, qu'il s'agit pour Stiegler de remettre en question le statut de "philosophie première » que Simondon, à la suite de Bergson, attribuait à la philosophie de la nature comprise comme cosmogenèse (Bergson) ou "ontogenèse" (Simondon). Nous voudrions ici exposer puis questionner cette opération critique de Stiegler, en procédant en trois temps :

- Rappeler brièvement la spécificité de l'ontogenèse simondonienne en ce qu'elle s'ancre dans des "schèmes physiques » qui informent l'hypothèse d'un état " préindividuel » de l'être, dont procède selon lui toute individuation (physique, vitale ou psychosociale) comprise comme genèse.

- Exposer les raisons pour lesquelles Stiegler est fondé à reprocher à Simondon de ne pas avoir pleinement pensé, à partir des travaux d'André Leroi-Gourhan, les conditions originellement techniques du dernier des «régimes d'individuation », à savoir le régime psycho-social ou «transindividuel».

- Discuter, enfin, le pas supplémentaire que fait Stiegler après cette critique, et qui consiste à soutenir que la pensée du transindividuel désormais «prothétiquement» fondé doit ellemême devenir la problématique première, à la manière dont Martin Heidegger pensait le Dasein avant même de penser les êtres vivants. Ce pas supplémentaire de Stiegler, et sa discussion par nous, engagent ainsi ce que nous nommons la «question du préindividuel », puisque Stiegler affirme en définitive «la dimension intrinsèquement technologique du préindividuel ». 


\section{Le rôle de l'hypothèse du préindividuel dans la théorie de l'individuation}

2 Vincent Bontems. Au risque de rappeler des éléments bien connus de la pensée de Gilbert Simondon, commençons par restituer le cadre général de sa théorie de l'individuation. Celle-ci repose en premier lieu sur le postulat (métaphysique) du « réalisme de la relation", qui accorde non seulement valeur d'être aux relations antérieurement aux termes qui se constituent au sein de ces relations, mais qui accorde, en outre, valeur d'être à une relation élaborée entre deux relations ayant elles-mêmes valeur d'être. Cette méthode analogique de construction s'applique en premier lieu aux sciences elles-mêmes, qui représentent des systèmes de relations plus ou moins stabilisées entre les relations expérimentalement observées et les relations mathématiques élaborées. Ceci explique la facilité avec laquelle Simondon incorpore les schèmes et concepts scientifiques comme d'authentiques expériences de pensée capables d'informer ou de réformer la pensée philosophique. Plus précisément, les schèmes physiques des rapports de phases et de la métastabilité thermodynamique qui viennent informer ce que Simondon nomme l'« hypothèse de l'état préindividuel de l'être »: contre le substantialisme mais aussi l'hylémorphisme de la tradition philosophique occidentale, il s'agit d'expliquer la genèse de toute réalité individuée en partant d'un état qui ne préfigurerait pas l'individualité comme le faisaient encore les « matière » et « forme » de l'hylémorphisme en tant qu'elles préexistaient à leur union. L'état préindividuel de l'être est alors un "potentiel réel », qui ne ne se réduit pas au possible abstrait, ni à un système d'actualités.

3 L'ontologie relationnelle ainsi constituée permet d'échapper aux apories des ontologies substantialistes traditionnelles, qui sont devenues de plus en plus impraticables au fur et à mesure des progrès des sciences de la nature, et que l'on peut juger définitivement caduques avec l'avènement de la mécanique quantique (comme le suggérait déjà Bachelard, et comme la critique du « réalisme des accidents » par Bernard D'Espagnat l'a bien montré). Dans cette perspective non-substantialiste, l'ontologie simondonienne des phénomènes physiques, biologiques et psychosociaux qui émerge est fondamentalement processuelle: le verre posé sur la table n'est plus une réalité statique, non seulement il est issu d'un processus technique de transformation, mais, en outre, sa nature amorphe signifie qu'à la différence du cristal, à une autre échelle de temps, il ne cesse d'évoluer, de couler très très lentement. Par là s'indique une autre caractéristique de la théorie de l'individuation : son matérialisme non-réductionniste car énergétiste et néoténique. Si toute individuation physique aboutit finalement à la dissipation des potentiels, l'individuation vitale consiste en une suspension de l'individuation physique, en un prolongement de sa phase inchoative, durant laquelle les échanges énergétiques avec le milieu permettent à l'individu de conserver certains potentiels, avant de mourir, c'est-à-dire de retourner à des échanges purement physiques avec son milieu. La même logique de rétention des potentiels explique l'émergence néoténique de l'individuation psychosociale à partir de l'individuation vitale.

4 Le schéma général de l'individuation est donc le suivant : l'individuation, qu'elle soit genèse radicale de l'individu physique ou vivant ou qu'elle soit perpétuation et « individualisation » de l'individu vivant déjà individué, consiste en un déphasage des potentiels en individu et son milieu associé avec lequel il entretient des relations 
énergétiques qui déterminent l'actualisation (partielle ou complète) de ses potentiels. L'originalité de cette théorie de l'individuation est de souligner la relativité de toute individuation aux ordres de grandeur: «En même temps qu'une énergie potentielle (condition d'ordre de grandeur supérieur) s'actualise, une matière s'ordonne et se répartit (condition d'ordre de grandeur inférieur) en individus structurés à un ordre de grandeur moyen, se développant par un processus médiat d'amplification ${ }^{1}$ ». C'est dans ce cadre que Simondon précise, dès l'Introduction de L'individuation à la lumière des notions de forme et d'individuation, ce qu'il désigne comme "l'hypothèse du préindividuel »: «on peut faire une hypothèse, analogue à celle des quanta en physique, analogue aussi à celle de la relativité des niveaux d'énergie potentielle : on peut supposer que l'individuation n'épuise pas toute la réalité préindividuelle, et qu'un régime de métastabilité est non seulement entretenu par l'individu, mais porté par lui, si bien que l'individu constitué transporte avec lui une certaine charge associée de réalité préindividuelle, animée par tous les potentiels qui la caractérisent ${ }^{2}{ } »$. La question qui se pose immanquablement est de savoir si la préindividualité est toujours relative à un ordre de grandeur supérieur ou s'il existe un domaine absolu de préindividualité, autrement dit une échelle à partir de laquelle il n'est plus possible de parler d'individus actuels. C'est ce qui explique la référence à la mécanique quantique : là où les autres philosophes rencontrent un problème, à savoir la disparition d'entités dotées d'une individualité permanente, Simondon trouve une solution inventive : le substantialisme devient radicalement impossible à l'échelle de Planck et doit laisser place au réalisme des relations. Au sens strict, le préindividuel désigne ainsi le soubassement ontologique que la théorie de l'individuation trouve dans la réalité quantique.

5 Observons alors qu'à cette échelle inframoléculaire, il n'y a pas de sens à distinguer, pour Simondon, entre matière inorganique, organique ou organisée, donc entre l'inerte, le vivant et le technique. La nature indécidable des "prions» rendus tristement populaires par l'épidémie de la vache folle illustre ce fait. Les développements récents des nanotechnologies pourraient sans doute amener à complexifier notre perception de l'individiduation et des rapports entre nature et artefact à ces échelles. Il y a là certainement matière à réactualiser l'approche, toujours pertinente, de Simondon sur la relativité de la validité de nos concepts en fonction de l'échelle à laquelle ils s'appliquent. Quoi qu'il en soit, il ne fait pas de doute que Stiegler n'a pas en vue cette préindividualité radicale quand il affirme le caractère « intrinsèquement technologique » du préindividuel. Il vise bien plutôt à circonscrire l'ensemble des potentiels rendant possible l'individuation transindividuelle des êtres humains. Avant même d'interroger cette démarche, et de céder la parole à mon camarade Jean-Hugues Barthélémy, j'aimerais souligner que ce geste n'est pas légitime dans l'économie de la philosophie simondonienne: non seulement, il convient de ne pas perdre de vue les conditions physiques et biologiques de l'individuation quant on aborde la question de l'individuation psychosociale, même si l'émotion joue pour l'individuation psychique et collective un rôle analogue à la préindividualité pour l'individuation physique, mais, surtout, la perspective de Simondon reste attachée, pour des raisons à la fois théoriques et axiologiques, à ne jamais isoler l'être humain du reste du règne animal. Elle refuse la "coupure anthropologique» jusque dans sa conception de la technique qu'elle étend, pour une part, à l'ensemble des êtres vivants (des insectes, dont le corps recèle des outils, aux grands primates, qui en confectionnent). La pensée de Simondon est en ce sens fidèle à son orientation 
matérialiste: d'abord cosmique, puis éthologique, avant de poser la question des rapports de l'homme à la machine.

\section{De Simondon à Stiegler : la refondation artefactuelle ou « prothétique » du transindividuel}

Jean-Hugues Barthélémy. L'œuvre de Bernard Stiegler entretient avec la pensée de Simondon un dialogue qui peut se résumer à ce que je nommerai la question du caractère non-anthropologique de la pensée de la technique. En effet, la «nonanthropologie" simondonienne n'a pas pour seul sens de refuser la coupure anthropologique entre l'homme et l'animal. Elle a aussi pour sens de refuser la coupure entre la réalité technique et la culture comme réalité humaine. Plus précisément, la non-anthropologie est ici dite telle parce qu'elle remet d'abord en question la réduction de la technique à un ensemble de moyens pour le travail humain, Simondon voulant au contraire penser le travail comme une partie de la technique, et le progrès technique comme un progrès du fonctionnement qui aurait ses propres lois et serait irréductible à tout usage de l'objet par l'homme. Or, c'est sur ce second terrain que Stiegler radicalise la non-anthropologie. En effet, s'il reconnaît bien à Simondon le mérite immense d'avoir ainsi montré que la fameuse aliénation machinique du travailleur tient en fait à ce que la culture n'a pas encore délégué à la machine le statut d'" individu technique » qu'avait autrefois le travailleur en tant que "porteur d'outils", il lui reproche cependant d'avoir fait de la technique une simple "phase» de la culture au lieu d'en faire ce à travers quoi les psychismes se sont toujours constitués en réalité psycho-sociale.

7 De fait, une faiblesse évidente de l'ouvrage de Simondon Du mode d'existence des objets techniques est que dans la troisième et dernière partie de cet ouvrage, Simondon développe une théorie des "phases de la culture " qui prétend penser le «stade magique » sans faire intervenir la réalité technique. Certes, ce que cet ouvrage entend par «objet technique" est sans doute bien plus étroit que la technique prêtée par Simondon aux animaux, et plus étroit aussi que la réalité technique pensée par Stiegler en tant qu'artefact en général. Mais ce que Simondon dit de ce qu'il nomme l' "unité magique primitive» ne fait pas non plus intervenir les artefacts, et privilégie des lieux naturels en tant que "points-clefs ", tel le sommet d'une montagne. Avant de préciser la difficulté, il faut ici rappeler que l'idée d' "unité » magique primitive tient à ce que Simondon pense le stade magique comme le lieu originel d'un déphasage qui donnera les phases culturelles de la "technique » et de la "religion », chacune de celles-ci se déphasant à son tour. L'art, lui, est alors autre chose qu'une phase proprement dite, puisqu'il se situe entre la religion et la technique et propose une restauration, certes toujours imparfaite, de l'unité primitive perdue.

Derrière la beauté de cette théorie des phases de la culture, qui ferait penser à certains égards à la théorie hégélienne de l'esprit objectif mais en remplaçant la dialectique par le déphasage "transductif» au sens donné par Simondon à ce terme, se pose donc le problème de l'absence des artefacts dans l'unité magique primitive. Que Simondon donne à la notion d'objet technique un sens suffisamment étroit pour faire ici de la technique une réalité qui se distingue radicalement de la religion à partir du déphasage de l'unité magique au lieu d'être constitutive de cette même religion, cela n'explique cependant pas que les artefacts au sens large soient absents de l'origine de la culture au 
profit des seuls «points-clefs» naturels. Stiegler pouvait donc bien, dans son article "Chute et élévation. L'apolitique de Simondon », reprocher à Simondon de supposer,

tout comme chez Rousseau, qu'il existe une humanité magique pré-technique : l'unité magique, c'est en effet ce qui, à l'exception de ces points clés, n'a pas encore détaché analytiquement du fond les formes, ou plutôt les schèmes, qui deviennent, comme outils techniques, des objets amovibles [...]. Ces thèses supposent donc que l' amovibilité technique, par laquelle Leroi-Gourhan, au contraire de Simondon, définit le processus d'individuation psychique et collective (couramment appelé l'homme) comme processus d'extériorisation, est ce qui survient comme déséquilibre et rupture de l'unité magique, tout à fait selon le schéma de Rousseau ${ }^{3}$.

Comme je l'ai montré par le menu, dans Penser l'individuation mais aussi dans Penser la connaissance et la technique après Simondon, la thèse stieglerienne du caractère " prothétique » du transindividuel en général peut se déduire d'une critique interne de la pensée simondonienne. Pour le dire ici en quelques phrases, on sait d'abord que Simondon, dans L'individuation à la lumière des notions de forme et d'information, nomme « sujet » l'animal en tant qu'il agit, qu'il perçoit et qu'il a des émotions. Le « sujet » animal est aussi considéré par Simondon comme l'ensemble formé par l'individu et sa " charge » de réalité préindividuelle. Or, l'affectivité est pour Simondon le lieu de cette charge préindividuelle, et l'émotion est pour cette raison ce qui peut engendrer une désindividuation provisoire de l'individu, comme rongé par son "potentiel préindividuel affectif ». Dès lors, si le sujet ne se laisse pas pour autant emporter par l'angoisse, il peut passer à l'individuation de son potentiel préindividuel affectif en personnalité psychosociale ou transindividuelle. Ainsi, Simondon est explicitement à la recherche de la médiation qui permettrait de rendre compte, dans ce passage du sujet psychique ou psycho-somatique à la personnalité psycho-sociale ou transindividuelle, de ce qu'il nomme l'« extériorité de l'intérieur » et l'« intériorité de l'extérieur »en parlant de cette personnalité transindividuelle: «il y a une antériorité du transindividuel par rapport à l'individuel qui empêche de définir un rapport de transcendance ou d'immanence ${ }^{4}$ ».

10 C'est précisément en ce point que Du mode d'existence des objets techniques apporte une solution sans pour autant, tel est le paradoxe, s'articuler au premier ouvrage ni approfondir sa réflexion nouvelle pour en faire une solution. Car Simondon y repensera embryonnairement le transindividuel à la lumière d'une technique qui était absente, elle, du premier ouvrage, et il fera presque de l'artefact ce lieu « antérieur » qui rend possible, dans la transindividualité psycho-sociale, l'intériorité de l'extérieur et l'extériorité de l'intérieur. Je dis "presque », parce qu'il s'arrêtera à l'idée que l'objet technique, et non l'artefact en général, est le « support » d'une relation humaine qui est « modèle de la transindividualité ${ }^{~ » . ~ P o u r t a n t, ~ i l ~ f e r a ~ b i e n ~ d e ~ l ' o b j e t ~ t e c h n i q u e ~ l ' e x p r e s s i o n ~}$ du préindividuel contenu dans le « sujet » lorsqu'il s'individue transindividuellement :

L'objet qui sort de l'invention technique emporte avec lui quelque chose de l'être qui l'a produit, exprime de cet être ce qui est le moins attaché à un hic et nunc; on pourrait dire qu'il y a de la nature humaine dans l'être technique, au sens où le mot de nature pourrait être employé pour désigner ce qui reste d'originel, d'antérieur même à l'humanité constituée en l'homme ${ }^{6}$.

11 Ces derniers mots sont à vrai dire typiques de ceux qui inciteront Bernard Stiegler à revendiquer un dépassement interne de Simondon même lorsqu'il s'agira pour lui de soutenir qu'il y a une « dimension intrinsèquement techno-logique du pré-individuel ». Mais avant de nous demander s'il est nécessaire d'aller jusque-là, on doit reconnaître mais aussi préciser que les derniers mots cités de Simondon incitent tout au moins mais 
aussi tout au plus à faire de la technique ce qui, chez le «sujet » animal, rend possible son individuation transindividuelle.

\section{La « question du préindividuel » suscitée par le refus d'une philosophie de la nature}

Dans son livre sur Les origines animales de la culture, Dominique Lestel reproche à Stiegler de retomber dans une coupure anthropologique en faisant de la technique le propre de l'homme. En ce sens, la radicalisation par Stiegler du caractère non-anthropologique de la pensée de la technique, celle-ci étant désormais constitutive de l'homme autant que constituée par lui, se ferait au détriment du caractère non-anthropologique de la pensée de l'homme comme issu du vivant. Or, s'il est vrai que les textes de Stiegler sont d'une ambiguïté suffisante pour se prêter à un tel reproche, je ne veux pas entrer ici dans ce débat, et aborderai bien plutôt ce qui me semble être la raison profonde de cette ambiguïté mais qui engage en fait une tout autre discussion : celle portant sur la nature de la problématique première en philosophie, pour laquelle Stiegler redéfinit en définitive le préindividuel. En effet, il est apparu que là où Simondon fait de la technique un prolongement du vivant et de l'objet technique un simple "support» d'une relation humaine qui est "modèle de la transindividualité », Stiegler fait de l'artefact le fondement même du transindividuel en général. Or, si ce transindividuel désormais "prothétiquement» fondé est tendanciellement identifié par Stiegler à l'homme, ce n'est pas seulement parce que Stiegler, contrairement à ce que semble comprendre de lui Lestel, pense des artefacts devenus «béquilles de l'esprit » inséparables d'un langage lui-même spécifique car historique, mais c'est aussi parce que plus fondamentalement le geste stieglerien se veut réflexif et «critique » au sens kantien : il s'agit, au moins projectivement, de penser les conditions techniques de la connaissance et donc de toute philosophie de la nature établie par ce qui est «toujours déjà » l'homme.

Telle est en définitive la grande rupture avec Simondon : Stiegler refuse de faire de la théorie de la connaissance ce qui procéderait d'une pensée du transindividuel dérivant elle-même d'une philosophie de la nature considérée comme " philosophie première ». Or, si j'ai dit que Stiegler avait « au moins projectivement » cette perspective réflexive et même critique, c'est parce que dans les faits le tome 3 de la Technique et le temps ne fait qu'annoncer une "nouvelle critique", et la démarche de Stiegler s'apparente en définitive davantage, à mon sens, à celle de Heidegger qui accorde au Dasein d'être le seul étant s'interrogeant sur son être. En effet cette démarche n'est ni proprement simondonienne ni proprement kantienne, mais revisite l'être-au-monde heideggerien comme ce qui ne s'ancre dans aucune philosophie de la nature mais doit pouvoir engendrer une théorie de la connaissance sans déjà la fournir par lui-même. Le propre de Stiegler serait alors, contre son ancien maître Jacques Derrida, de délaisser le Heidegger de la Kehre pour revenir à Sein und Zeit et y introduire une dimension artefactuelle de la Weltgeschichtlichkeit : «1. Le Dasein, essentiellement factice, est prothétique. Il n'est rien hors de ce qui est hors de lui ni de ce qu'il est hors de lui, car c'est là par là seulement qu'il éprouve, sans jamais la prouver, sa mortalité, qu'il l'anticipe. 2. L' accès du Dasein à son passé [...] est aussi pro-thétique. C'est selon une telle condition qu'il accède ou n'accède pas à ce passé tel qu'il a été ou non durablement fixé, et à quoi, du

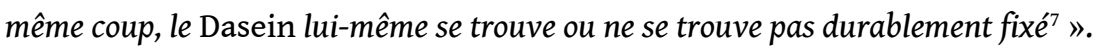


Stiegler peut certes se revendiquer de Simondon lui-même lorsqu'il rappelle que la connaissance de l'individuation est elle-même individuation de la connaissance. Mais la question se pose de savoir si cette fidélité revendiquée à Simondon se maintient lorsque Stiegler en conclut que le préindividuel ne peut plus aujourd'hui être pensé que comme toujours déjà « intrinsèquement technologique ». Stiegler profite de ce que Simondon a pu écrire que le transindividuel « est cette réalité que l'être individué transporte avec lui, cette charge d'être pour des individuations futures ${ }^{8} »$. Ici en effet le préindividuel et le transindividuel semblent se confondre. Mais c'est parce que l'individuation transindividuelle ou psycho-sociale ne reconduira plus jamais au simple individu, puisqu'étant rendue possible par le «sujet» comme ensemble individu-charge préindividuelle, et conduisant à une "personnalité » pour laquelle le social n'est plus un simple milieu associé mais une réalité elle-même individuée possédant sa propre personnalité, qui lui est «coextensive»: "Ce n'est pas véritablement en tant qu'individus que les êtres sont rattachés les uns aux autres dans le collectif, mais en tant que sujets, c'est-à-dire en tant qu'êtres qui contiennent du pré-individuel ${ }^{9}$ ".

Si donc la fidélité de Stiegler à Simondon prend fin ici, est-il maintenant nécessaire de soutenir que le préindividuel ne peut plus aujourd'hui être pensé que comme toujours déjà « intrinsèquement technologique » et transindividualisant ? Dans l'article déjà cité, Stiegler entend de fait, cette fois, dépasser Simondon en écrivant ce passage décisif, dont le commentaire critique achèvera nos réflexions : «il y a un éternel retour de l'individu transindividué au stade pré-individuel où le transindividuel redevient un matériau pulsionnel (et non seulement instinctuel). Or, ce qui constitue ce circuit, c'est la technicité de l'individuation. La modalité techno-logique de l'inachèvement, que l'on appelle, depuis le $\mathrm{xx}^{\mathrm{e}}$ siècle, l'existence, est ce qui constitue l'individuation psychosociale en tant qu'elle individue un potentiel pré-individuel sursaturé non plus comme devenir et ontogenèse d'une espèce vivante, mais bien comme co-individuation d'un individu psychique et d'un groupe social à l'intérieur duquel il s'individue en y provoquant un processus de résonance interne où s'individue aussi l'ensemble des éléments techniques à travers les individus techniques ${ }^{10} »$.

16 "Eternel retour », «existence »: ces mots ne sont pas innocents mais laissent au contraire deviner que Stiegler, au sein d'un dépassement de Simondon qui s'annonçait dans le tome 3 de La technique et le temps comme possédant une finalité « réflexive et critique » au sens kantien, se dirige en fait davantage, via ses premiers travaux sur Heidegger puis ses travaux actuels sur Sigmund Freud, vers ce que je nommerai une «ontologie fondamentale nietzschéenne » qui se substituerait à la philosophie de la nature en court-circuitant toute histoire du préindividuel menant au transindividuel. Sa force est alors de pouvoir invoquer le fait que Simondon lui-même disait les « phases de l'être » non-successives. Mais la question se pose de savoir comment rendre viable la thèse, présente en ce texte comme en d'autres, selon laquelle la technique serait à la fois ce qui s'individue comme " troisième brin » de l'individuation psychique et collective et ce qui constitue le fonds pré-individuel de cette individuation psychique et collective.

17 Vincent Bontems. J'embraye ici sur la caractérisation par Barthélémy du dépassement stieglerien de Simondon en direction d'une nouvelle "psychologie-sociologie des profondeurs » plutôt nietzschéène en soulignant que c'est précisément à propos de la question du transindividuel que Simondon faisait appel à la figure de Zarathoustra. Cette référence intervient dans un passage qui évite précisément de penser les 
conditions technologiques de l'individuation psychosociale, et privilégie, au contraire, une formulation religieuse en termes d'immanence et de transcendance :

si l'on admet que le transindividuel est auto-constitutif, on verra que le schème de transcendance ou le schème d'immanence ne rendent compte de cette autoconstitution que par leur position simultanée et réciproque : c'est en effet à chaque instant de l'autoconstitution que le rapport entre l'individu et le transindividuel se définit comme ce qui dépasse l'individu tout en le prolongeant ${ }^{11}$.

L'interprétation stieglerienne consiste alors à tra(ns)duire cette problématique de l'autoconstitution, exprimée par Simondon selon les schèmes de la phase religieuse, dans les termes de la phase technique, en tirant parti du fait que la réalité prothétique de la technique constitue précisément «ce qui dépasse l'individu tout en le prolongeant » du point de vue matériel. En ce sens, il s'agit bien d'un geste correctif éminemment fidèle à l'inspiration matérialiste de la théorie de l'individuation.

Pour autant, ce geste n'est pas exempt de risques de dérives idéalistes dans la mesure même où il court-circuite la pluralité irréductible du système des phases. En effet, en faisant du développement technologique l'unique modalité de l'être qui «se préexiste à travers son présent ${ }^{12} "$, Stiegler instaure une rupture avec la dimension cosmique de l'être-au-monde qu'incarne justement Zarathoustra quand il remonte en haut de la montagne après la mort de son ami le danseur de corde. Car, s'il y a un oubli de l'artefact et du fétiche dans la conception de la pensée magique chez Simondon, il y a aussi le risque, à se focaliser seulement sur l'extériorité de l'artefact, d'oublier l'inscription géographique du réseau magique, des points-clefs et des voults. La disparition des forêts vierges et des banquises constitue à ce titre une catastrophe aussi considérable, et bien plus irrémédiable, pour notre capacité à aimer le monde et à lui donner sens et valeur que la pollution audiovisuelle qui détermine selon Stiegler le devenir «immonde» de notre existence. Aussi paradoxal que cela puisse paraître, l'attention exclusive portée à l'évolution des conditions technologiques de l'existence humaine conduit à une forme d'idéalisme, puisqu'elle met en avant l'irréversibilité temporelle d'un processus dont il faudrait interroger en permanence le déploiement dynamique dans l'espace. Dans cette perspective, les analyses stiegleriennes sont souvent reconduites à l'exposition d'un devenir linéaire, tout comme Karl Marx, bien qu'étant plus que tout autre sensible aux structures réelles et géopolitiques de son temps, se trouvait, par le jeu de la dialectique, amené à exprimer sa pensée dans une perspective purement historique.

Comme je l'ai montré dans mon article sur "Encyclopédisme et crise de la culture ", Simondon défend dans son "Introduction au projet d'encyclopédie génétique » une conception " polyphasée » du progrès. Après avoir connu des phases philosophique et scientifique, le progrès est à notre époque essentiellement d'ordre technologique, mais le défi qui s'ouvre devant nous est de relativiser le sens de chacune de ces phases et non de vouloir trouver dans la dernière étape la vérité des deux premières. Qui plus est, la crise actuelle entre la culture spirituelle et la civilisation technique ne survient selon Simondon qu'en raison d'une rupture d'échelle entre l'extension des réseaux des valeurs culturelles et les réseaux des valeurs technologiques: des hommes qui ne partagent plus aucune valeur utilisent pourtant les mêmes artefacts technologiques. Cette épistémologie des échelles qui travaille toute la pensée de Simondon inscrit toujours les processus dans une réalité spatio-temporelle, historique et géographique, qui rend caduque les schémas évolutifs linéaires et la synchronie absolue. 
De toutes ces évolutions et crises, Stiegler est l'un des observateurs les plus attentifs et les plus inspirés. Reste qu'il marque justement sa différence avec Simondon d'une manière qui témoigne que c'est encore Simondon qui permet le mieux de comprendre la situation dans laquelle il se trouve. Tel est le paradoxe de l'autoconstitution du transindividuel, que Barthélémy nous a appris à comprendre comme "autotranscendance du sens ", et qui nous interdit, selon moi, de croire en la simplicité des « dépassements » successifs ou de "l'unité spirituelle » d'une époque : la préindividualité reste d'actualité. En discutant Simondon à la lumière de Heidegger ou Freud et, au moins téléologiquement, de Nietzsche, c'est-à-dire dans le prisme des interprétations multiples et relatives, Stiegler nous donne ainsi aussi les moyens de le discuter, lui, à la lumière de Simondon.

\section{BIBLIOGRAPHIE}

Simondon Gilbert, Du mode d'existence des objets techniques, Paris, Aubier, 1958.

Simondon Gilbert, L'individuation à la lumière des notions de forme et d'information, Grenoble, Millon, 2005.

Stiegler Bernard, La technique et le temps, t. I, Paris, Galilée, 1994.

Stiegler Bernard, «Chute et élévation. L'apolitique de Simondon », Revue philosophique, $\mathrm{n}^{\circ}$ 3, 2006.

\section{NOTES}

1. Gilbert Simondon, L'individuation à la lumière des notions de forme et d'information, Grenoble, Millon, 2005, p. 27.

2. Ibid., p. 28.

3. Bernard Stiegler, «Chute et élévation. L'apolitique de Simondon », Revue philosophique, $\mathrm{n}^{\circ} 3$, 2006, p. 333.

4. Gilbert Simondon, L'individuation à la lumière des notions de forme et d'information, p. 304.

5. Du mode d'existence des objets techniques, Paris, Aubier, 1958, p. 247-248.

6. Ibid.

7. Bernard Stiegler, La technique et le temps, t. I, Paris, Galilée, 1994, p. 240.

8. Gilbert Simondon, L'individuation à la lumière des notions de forme et d'information, p. 303.

9. Ibid., p. 310.

10. Bernard Stiegler, « Chute et élévation », p. 327-328.

11. Gilbert Simondon, L'individuation à la lumière des notions de forme et d'information, p. 381.

12. Ibid., p. 288. 


\section{AUTEURS}

\section{JEAN-HUGUES BARTHÉLÉMY}

Jean-Hugues Barthélémy est docteur en épistémologie et histoire des sciences et techniques (Université Paris 7 - Denis Diderot). Il a dirigé le séminaire « Simondon ou l'Encyclopédisme génétique » de la Maison des sciences de l'Homme de Paris-Nord entre 2006 et 2008. Il est l'auteur de trois ouvrages sur Simondon, dont le dernier est paru en mai 2008 aux P.U.F. : Simondon ou l'Encyclopédisme génétique.

\section{VINCENT BONTEMS}

Laboratoire de Recherche sur les Sciences de la Matière (LARCIM-CEA) 